H. Tamura

Nagoya Math. J.

Vol. 60 (1976), 7-33

\title{
THE ASYMPTOTIC DISTRIBUTION OF EIGENVALUES OF THE LAPLACE OPERATOR IN AN UNBOUNDED DOMAIN
}

\author{
HIDEO TAMURA
}

\section{§ 1. Introduction.}

This paper is devoted to the study of the asymptotic distribution of eigenvalues of the Laplace operator with zero boundary conditions in a quasi-bounded domain contained in Euclidean space $\boldsymbol{R}^{2}$. Let us consider the following eigenvalue problem:

$$
-\left(\frac{\partial^{2}}{\partial x_{1}^{2}}+\frac{\partial^{2}}{\partial x_{2}^{2}}\right) u=\lambda u, \quad u \in H_{0}^{1}(\Omega),
$$

where

$$
\Omega=\left\{\left(x_{1}, x_{2}\right) \mid-\infty<x_{1}<\infty, 0<x_{2}<q\left(x_{1}\right)\right\},
$$

and $q(x)$ is a smooth positive function defined on $(-\infty, \infty)$ satisfying $\lim _{|x| \rightarrow \infty} q(x)=0$.

It has been shown in [1] that the problem (1.1) has an infinite sequence of discrete eigenvalues approaching to $\infty$. We denote by $N(h)$ the number of eigenvalues less than $h$ of the problem (1.1). We are concerned with the asymptotic behavior of $N(h)$ as $h \rightarrow \infty$.

The asymptotic distribution of eigenvalues of the Laplace operator with zero boundary conditions in a quasi-bounded domain has been studied by Clark [1], Hewgill [4], and Glazman and Skacek [2]. It seems to the author that any true asymptotic formula for $N(h)$ even in the case of such a simple domain as (1.2) has not been known.

We shall study the problem (1.1) under the formulation as an eigenvalue problem of a differential operator with operator-valued coefficients. On the other hand, Kostjuchenko and Levitan [5] studied the eigenvalue problem for the operator $-\left(d^{2} / d t^{2}\right)+Q(t)$ under the assumption that $Q(t)$ is

Received April 30, 1975. 
a semi-bounded self-adjoint operator for each fixed $t \in R^{1}$ with the common domain of definition $\mathscr{D}(Q(t))=\mathscr{D}_{0}$ and other restrictions. Our method is different from that in [5] in some ways.

DEFINITION 1.1. We denote by $K(m)(0<m \leq 1)$ the set of smooth functions $q(x)$ defined on $\boldsymbol{R}^{1}$ satisfying the following conditions: There exist positive constants $C_{1}, C_{2}$ and $C$ independent of $x$ and $y$ such that

(i ) $C_{1}(1+|x|)^{-m} \leq q(x) \leq C_{2}(1+|x|)^{-m}$,

(ii) for $|x-y| \leq 1,|q(x)-q(y)| \leq C q(x)|x-y|$,

(iii) $\left|\left(\frac{d}{d x}\right)^{j} q(x)\right|=\left|q^{(j)}(x)\right| \leq C q(x) \quad(j=1,2)$.

Now we shall state our main theorem which will be proved in $\S 4$.

THEOREM 1.1. Let $\left\{\zeta_{j}>0\right\}_{j=1}^{\infty}$ be eigenvalues of the operator $A_{0}$ $\left(=-\frac{\partial^{2}}{\partial y^{2}}\right)$ with the domain of definition $\mathscr{D}\left(A_{0}\right)=H_{0}^{1}(0,1) \cap H^{2}(0,1)$. Suppose that $q(x)$ belongs to $K(m)(0<m \leq 1)$. Then, as $h \rightarrow \infty$

$$
N(h) \sim \frac{1}{\pi} \sum_{j=1}^{\infty} \int_{\Omega_{j}(h)}\left(h-\zeta_{j} q(x)^{-2}\right)^{1 / 2} d x,
$$

where $\Omega_{j}(h)=\left\{x \in \boldsymbol{R}^{1} \mid h q(x)^{2}>\zeta_{j}\right\}$, while $\left.f(h)\right) \sim g(h)$ means that $\lim _{h \rightarrow \infty} f(h)^{-1} g(h)=1$.

Remark. $\left\{\zeta_{j} q(x)^{-2}\right\}_{j=1}^{\infty}$ are regarded as eigenvalues of the operator $-\frac{\partial^{2}}{\partial y^{2}}$ with the domain of definition $H_{0}^{1}(0, q(x)) \cap H^{2}(0, q(x))$.

EXAMPLE. If $\lim _{|x| \rightarrow \infty}|x|^{m} q(x)=a(>0)$, then

$$
\begin{aligned}
N(h) & \sim C(a) h^{-1 / 2+1 / 2 m} & & (0<m<1), \\
& \sim C(a) h \log h & & (m=1) .
\end{aligned}
$$

Throughout this paper, we confine ourselves to such a simple problem as (1.1), but some generalizations will be discussed without proofs in §7. Finally we note that in this paper we use one and the same symbol $C$ in order to denote positive constants which may differ from each other. When we specify the dependence of such a constant on a parameter, say $m$, we denote it by $C(m)$ or $C_{m}$. 


\section{§ 2. Preliminaries.}

Let us introduce some function spaces.

$H^{j}(0,1)$ and $H_{0}^{j}(0,1)(j=1,2, \cdots)$ denote the usual Sobolev spaces on the interval $(0,1)$. For any real $s$, the Sobolev space $H^{s}(0,1)$ can be defined by interpolation methods ([6]). We denote by $X$ the Hilbert space $L^{2}(0,1)$ with the usual scalar product $(u, v)_{0}=\int_{0}^{1} u(x) \overline{v(x)} d x$, and the norm $\|u\|_{0}=\int_{0}^{1}|u|^{2} d x . \quad L^{2}(-\infty, \infty ; X)$ denotes the Hilbert space of $X$ valued square integrable functions with the scalar product $\langle f, g\rangle=$ $\int_{-\infty}^{\infty}(f(x), g(x))_{0} d x$.

Let $q(x)$ be a function belonging to $K(m)$ and let $\Omega$ be an open domain defined by $\Omega=\left\{\left(x_{1}, x_{2}\right) \mid-\infty<x_{1}<\infty, 0<x_{2}<q\left(x_{1}\right)\right\}$ and $G$ be a cylinder domain defined by $G=\{(x, y) \mid-\infty<x<\infty, 0<y<1\}$. Then we define the unitary operator $U$ from $L^{2}(\Omega)$ onto $L^{2}(G)$ by

$$
(U \cdot u)(x, y)=q(x)^{1 / 2} u(x, q(x) y), \quad u \in L^{2}(\Omega) .
$$

Similary we define the unitary operator $V\left(=U^{*}\right)$ from $L^{2}(G)$ onto $L^{2}(\Omega)$ by

$$
(V \cdot v)\left(x_{1}, x_{2}\right)=q\left(x_{1}\right)^{-1 / 2} v\left(x_{1}, q\left(x_{1}\right)^{-1} x_{2}\right), \quad v \in L^{2}(G) .
$$

Now consider the following eigenvalue problem in $L^{2}(\Omega)$ :

$$
H u=-\left(\frac{\partial^{2}}{\partial x_{1}^{2}}+\frac{\partial^{2}}{\partial x_{2}^{2}}\right) u=\lambda u, \quad u \in H_{0}^{1}(\Omega) .
$$

Here the operator $H$ is a positive self-adjoint operator associated with the symmetric bilinear form

$$
a(u, v)=\int_{\Omega}\left(\frac{\partial}{\partial x_{1}} u \frac{\partial}{\partial x_{1}} \bar{v}+\frac{\partial}{\partial x_{2}} u \frac{\partial}{\partial x_{2}} \bar{v}\right) d x_{1} d x_{2}, \quad u, v \in H_{0}^{1}(\Omega) .
$$

By using the operators $U$ and $V$, we shall transform the above problem (1.1) into the problem in $L^{2}(G)$.

Let $U$ and $V$ be the operators defined by (2.1) and (2.2) respectively. Then, we have

$$
U \frac{\partial}{\partial x_{1}} V=\frac{d}{d x}-q(x)^{-1} q^{(1)}(x) y \frac{\partial}{\partial y}-\frac{1}{2} q(x)^{-1} q^{(1)}(x)=\frac{d}{d x}+P(x),
$$




$$
U \frac{\partial}{\partial x_{2}} V=q(x)^{-1} \frac{\partial}{\partial y}=Q(x)
$$

where for each fixed $t \in \boldsymbol{R}^{1}, P(t)$ and $Q(t)$ are regarded as operators acting on $X$ with the domain of definition $\mathscr{D}(P(t))=\mathscr{D}(Q(t))=H_{0}^{1}(0,1)$. Here we remark that the coefficients of the operator $P(t)$ are uniformly bounded. By using (2.4) and (2.5), the symmetric bilinear form $a(u, v)$ is transformed into a bilinear form

$$
\begin{aligned}
b(u, v)= & \int_{-\infty}^{\infty}\left(\left(\frac{d}{d x}+P(x)\right) u,\left(\frac{d}{d x}+P(x)\right) v\right)_{0} d x \\
& +\int_{-\infty}^{\infty}(Q(x) u, Q(x) v)_{0} d x
\end{aligned}
$$

where $b(u, v)$ is defined on the set $\mathscr{D}(b)=\left\{u \in L^{2}(-\infty, \infty ; X) \mid \frac{d}{d x} u \in L^{2}\right.$ $\left.\cdot(-\infty, \infty ; X), Q(x) u \in L^{2}(-\infty, \infty ; X)\right\}$. The symmetric bilinear form $b(u, v)$ induces a unique positive self-adjoint operator $T$ in the sense of Friedrichs. $T$ has the following expression:

$$
\begin{aligned}
T & =-\left(\frac{d}{d x}\right)^{2}-\frac{d}{d x} P(x)+P^{*}(x) \frac{d}{d x}+P^{*}(x) P(x)+Q^{*}(x) Q(x) \\
& =-\left(\frac{d}{d x}\right)^{2}-\frac{d}{d x} P(x)+P^{*}(x) \frac{d}{d x}+A(x)
\end{aligned}
$$

where for each fixed $t \in R^{1}, P^{*}(t)$ and $Q^{*}(t)$ denote the adjoint operators in $X$ and $A(t)$ ( $t$; fixed) is a self-adjoint operator with the domain of definition $\mathscr{D}(A(t))=H_{0}^{1}(0,1) \cap H^{2}(0,1)$. Thus we have transformed the eigenvalue problem (1.1) in $L^{2}(\Omega)$ into the following equivalent problem (2.8) in $L^{2}(-\infty, \infty ; X)$ :

$$
T u=-\left(\frac{d}{d x}\right)^{2} u-\frac{d}{d x} P(x) u+P^{*}(x) \frac{d}{d x} u+A(x) u=\lambda u .
$$

We denote by $\left\{\mu_{j}\right\}_{j=1}^{\infty}$ and $\left\{u_{j}\right\}_{j=1}^{\infty}$ eigenvalues of the problem (2.8) and the normalized eigenfunctions corresponding to $\left\{\mu_{j}\right\}_{j=1}^{\infty}$ respectively.

Let $A_{0}$ be the positive self-adjoint operator $-\frac{\partial^{2}}{\partial y^{2}}$ with the domain of definition $\mathscr{D}\left(A_{0}\right)=H_{0}^{1}(0,1) \cap H^{2}(0,1)$. Then, we have with a constant $C$ independent of $t \in R^{1}$, 


$$
\|A(t) u\|_{0} \leq C q(t)^{-2}\left\|A_{0} u\right\|_{0}, \quad \text { for any } u \in \mathscr{D}\left(A_{0}\right) \text {, }
$$

and

$$
\left\|A(t)^{1 / 2} v\right\|_{0} \geq C q(t)^{-1}\left\|A_{0}^{1 / 2} v\right\|_{0}, \quad \text { for any } v \in \mathscr{D}\left(A_{0}^{1 / 2}\right) .
$$

Hence, with the aid of the Heinz interpolation inequality, we have

LEMMA 2.1.

(2.9) $\left\|A(t)^{\alpha} u\right\|_{0} \leq C q(t)^{-2 \alpha}\left\|A_{0}^{\alpha} u\right\|_{0}, \quad$ for $u \in \mathscr{D}\left(A_{0}^{\alpha}\right),(0 \leq \alpha \leq 1)$,

(2.10) $\left\|A(t)^{\beta} u\right\|_{0} \geq C q(t)^{-\beta}\left\|A_{0}^{\beta} u\right\|_{0}, \quad$ for $u \in \mathscr{D}\left(A_{0}^{\beta}\right),(0 \leq \beta \leq 1 / 2)$,

where $C$ is a constant independent of $t \in \boldsymbol{R}^{1}$ and $u$.

The following lemma is obvious from the definitions of the operators $P(t)$ and $A(t)$.

LEMMA 2.2. For any $t$ and $s$ such that $|t-s| \leq 1$, we have

$$
\begin{aligned}
& \|(P(t)-P(s)) u\|_{0} \leq C|t-s|\left\|A(t)^{1 / 2} u\right\|_{0}, \\
& \left\|\left(P^{*}(t)-P^{*}(s)\right) u\right\|_{0} \leq C|t-s|\left\|A(t)^{1 / 2} u\right\|_{0}, \\
& \|(A(t)-A(s)) v\|_{0} \leq C|t-s|\|A(t) v\|_{0},
\end{aligned}
$$

where $C$ is a constant independent of $t, s, u \in \mathscr{D}\left(A(t)^{1 / 2}\right)=H_{0}^{1}(0,1)$ and $v \in \mathscr{D}(A(t))$.

By Lemma 2.2, we see that the operators $A(t)^{-1 / 2}(P(t)-P(s)), A(t)^{-1 / 2}$ . $\left(P^{*}(t)-P^{*}(s)\right)$ and $A(t)^{-1}(A(t)-A(s))$ can be extended to bounded operators in $X$ and satisfy

$$
\begin{gathered}
||\left|A(t)^{-1 / 2}(P(t)-P(s))\|\|_{0} \leq C\right| t-s \mid, \\
\left\||| A(t)^{-1 / 2}\left(P^{*}(t)-P^{*}(s)\right)\left|\|_{0} \leq C\right| t-s \mid,\right. \\
||\left|A(t)^{-1}(A(t)-A(s))\right| \|_{0} \leq C|t-s|, \quad(|t-s| \leq 1)
\end{gathered}
$$

where $\|\cdot\| \|_{0}$ stands for the usual operator norm for bounded operators in $X$.

LEMMA 2.3. Let $b(u, v)$ be the symmetric bilinear form defined by (2.6). Then, we have for a constant $C$ independent of $u \in \mathscr{D}(b)$,

$$
b(u, u) \geq C \int_{-\infty}^{\infty}\left\{\left(\frac{d}{d x} u, \frac{d}{d x} u\right)_{0}+|x|^{2 m}\left(A_{0}^{1 / 2} u, A_{0}^{1 / 2} u\right)_{0}\right\} d x .
$$


Proof. We note that there exist constants $C$ and $\beta$ independent of $x$ and $v \in \mathscr{D}\left(A_{0}^{1 / 2}\right)$ such that $\|P(x) v\|_{0}^{2} \leq \beta\|Q(x) v\|_{0}^{2}$ and $\|Q(x) v\|_{0}^{2} \geq C|x|^{2 m}$ $\cdot\left\|A_{0}^{1 / 2} v\right\|_{0}^{2}$. For any $\varepsilon>0$, we have

$$
\begin{aligned}
b(u, u) & \geq \int_{-\infty}^{\infty}\left(\left\|\frac{d}{d x} u\right\|_{0}^{2}-2\left\|\frac{d}{d x} u\right\|_{0}\|P(x) u\|_{0}+\|P(x) u\|_{0}^{2}+\|Q(x) u\|_{0}^{2}\right) d x \\
& \geq \int_{-\infty}^{\infty}\left(\varepsilon\left\|\frac{d}{d x} u\right\|_{0}^{2}-\varepsilon \beta /(1-\varepsilon)\|Q(x) u\|_{0}^{2}+\|Q(x) u\|_{0}^{2}\right) d x .
\end{aligned}
$$

Hence, by choosing $\varepsilon$ in $(2.15)$ so that $\varepsilon(1+\beta)<1$, we obtain the proof.

Q.E.D.

Let us consider the following eigenvalue problem in $L^{2}(-\infty, \infty ; X)$ :

$$
-\frac{d^{2}}{d x^{2}} u+|x|^{2 m} A_{0} u=\lambda u
$$

We denote by $\left\{\zeta_{j}\right\}_{j=1}^{\infty}$ eigenvalues of the operator $A_{0}$ and by $\left\{\lambda_{k}\right\}_{k=1}^{\infty}$ eigenvalues of the operator $-\frac{d^{2}}{d x^{2}}+|x|^{2 m}$ considered in $L^{2}(-\infty, \infty)$. Then with the aid of separation of variables, we easily see that the eigenvalues $\left\{\nu_{j}\right\}_{j=1}^{\infty}$ of the problem (2.16) are given by $\left\{\zeta_{j}^{1 /(1+m)} \lambda_{k}\right\}_{j, k=1}^{\infty}$.

LEMMA 2.4. Let $\left\{\nu_{j}\right\}_{j=1}^{\infty}$ be eigenvalues of the problem (2.16). Then, for any $p>1 / 2+1 / 2 m$ and $h>2$, we have

$$
\begin{aligned}
\sum_{j=1}^{\infty}\left(\nu_{j}+h\right)^{-p} & \leq C(p) h^{1 / 2+1 / 2 m-p} & & (0<m<1) \\
& \leq C(p) h^{1-p} \log h & & (m=1) .
\end{aligned}
$$

Proof. It is known that there exists a constant $C$ independent of $k$ such that $\lambda_{k} \geq C k^{2 m /(m+1)}$ and it is clear that $\zeta_{j} \geq C j^{2}$. ([7])

Hence, it follows that

$$
\sum_{j=1}^{\infty}\left(\nu_{j}+h\right)^{-p} \leq C \sum_{j, k=1}^{\infty}\left(j^{2 /(m+1)} k^{2 m /(m+1)}+h\right)^{-p} .
$$

Furthermore, by using the estimate with a constant $C$ independet of $h$

$$
\sum_{k=1}^{\infty}\left(k^{2 m /(m+1)}+h\right)^{-p} \leq C h^{1 / 2+1 / 2 m-p},
$$

we have

$$
\sum_{j, k=1}^{\infty}\left(j^{2 /(m+1)} k^{2 m /(m+1)}+h\right)^{-p} \leq C \sum_{j=1}^{\infty} j^{-1 / m} h^{1 / 2+1 / 2 m-p}
$$


This gives the proof in the case of $0<m<1$. When $m=1$, it is easy to see that $\sum_{j, k=1}^{\infty}(j k+h)^{-p} \leq C h^{1-p} \log h$, which completes the proof.

Q.E.D.

Proposition 2.1. Let $\left\{\mu_{j}\right\}_{j=1}^{\infty}$ be eigenvalues of the problem (2.8). Then, for any $p>1 / 2+1 / 2 m$ and $h>2$, we have with a constant $C$ independent of $h$,

$$
\begin{aligned}
\sum_{j=1}^{\infty}\left(\mu_{j}+h\right)^{-p} & \leq C h^{1 / 2+1 / 2 m-p} & & (0<m<1, \\
& \leq C h^{1-p} \log h & & (m=1) .
\end{aligned}
$$

Proof. By virture of Lemma 2.3, we see that there exists a constant $C$ independent of $j$ such that $\mu_{j} \geq C \nu_{j}$. Hence, by combining this fact with Lemma 2.4, we get our assertion.

Q.E.D.

\section{§ 3. Propositions.}

In this section, we shall state fundamental propositions which will be used later.

Let us fix some notations.

Let $Y$ be a separable Hilbert space with the scalar product $(,)_{Y}$ and the norm \|\|$_{Y} \cdot B(Y)$ stands for the Banach space of all bounded operators acting on $Y$ with the operator norm $\mid\|\cdot\| \|_{0}$, and $B_{\alpha}(Y)$ denotes the subspace of $B(Y)$ consisting of compact operators such that $\|K\| \|_{\alpha}=$ $\left(\sum_{j=1}^{\infty} \beta_{j}^{\alpha}\right)^{1 / \alpha}<\infty$, where $\left\{\beta_{j}>0\right\}_{j=1}^{\infty}$ denote eigenvalues of $\left(K^{*} K\right)^{1 / 2}$.

Proposition 3.1. Let $K(x)(-\infty<x<\infty)$ be a family of operators belonging to $B_{2}(Y)$ such that $K(x)$ is continuous under the norm $\|\cdot\| \|_{0}$ with respect to $x$ and that $\int_{-\infty}^{\infty}\|K(x)\|_{2}^{2} d x<+\infty$. Let $\left\{\psi_{j}(x\}_{j=1}^{\infty}\right.$ be a complete orthonormal system in $L^{2}(-\infty, \infty ; Y)$. If we define $\beta_{j}=$ $\int_{-\infty}^{\infty} K(x) \psi_{j}(x) d x$, then $\sum_{j=1}^{\infty}\left\|\beta_{j}\right\|_{Y}^{2}=\int_{-\infty}^{\infty} \mid\|K(x)\|_{2}^{2} d x$.

Proof. Let $\left\{\theta_{j}\right\}_{j=1}^{\infty}$ be a complete orthonormal system in $Y$. Then, we set $k^{j i}(x)=\left(K(x) \theta_{j}, \theta_{i}\right)_{Y}$ and $t_{j i}(x)=\left(\psi_{j}(x), \theta_{i}\right)_{Y}$. We note that $\left\{t_{j}(x)=\left(t_{j i}(x)\right)_{i=1}^{\infty}\right\}_{j=1}^{\infty}$ forms a complete orthonormal system in $L^{2}\left(-\infty, \infty ; \ell^{2}\right)$, where $\ell^{2}$ denotes the usual Hilbert space consisting of all complex-valued square summable series. By the above definitions of $k^{j i}(x)$ and $t_{j i}(x)$, we have 


$$
K(x) \psi_{j}(x)=\sum_{i, \ell=1}^{\infty} t_{j i}(x) k^{i \ell}(x) \theta_{\ell} .
$$

By (3.1), we obtain

$$
\begin{aligned}
\sum_{j=1}^{\infty}\left\|\beta_{j}\right\|_{Y}^{2} & =\sum_{j=1}^{\infty}\left(\int_{-\infty}^{\infty} K(x) \psi_{j}(x) d x, \int_{-\infty}^{\infty} K(x) \psi_{j}(x) d x\right)_{Y} \\
& =\sum_{j=1}^{\infty}\left(\sum_{i, \ell=1}^{\infty} \int_{-\infty}^{\infty} t_{j i}(x) k^{i \ell}(x) d x \theta_{\ell}, \sum_{n, m=1}^{\infty} \int_{-\infty}^{\infty} t_{j n}(x) k^{n m}(x) d x \theta_{m}\right)_{X} \\
& =\sum_{j=1}^{\infty} \sum_{\ell=1}^{\infty}\left|\sum_{i=1}^{\infty} \int_{-\infty}^{\infty} t_{j i}(x) k^{i \ell}(x) d x\right|^{2} \\
& =\sum_{i=1}^{\infty} \sum_{l=1}^{\infty} \int_{-\infty}^{\infty}\left|k^{i \ell}(x)\right|^{2} d x=\int_{-\infty}^{\infty} \mid\|K(x)\|_{2}^{2} d x,
\end{aligned}
$$

where we have used the fact that $\left\{t_{j}(x)\right\}_{j=1}^{\infty}$ forms a complete orthonormal system in $L^{2}\left(-\infty, \infty ; \ell^{2}\right)$. This completes the proof.

Let $\mathscr{H}_{r}(\gamma$ : positive real number) be the Hilbert space with the scalar product $(u, v)_{r}=\left(A_{0}^{r} u, A_{0}^{\tau} v\right)_{0}$ and the norm $\|u\|_{r}^{2}=\left\|A_{0}^{\tau} u\right\|_{0}^{2}$. Let $\mathscr{H}_{-\gamma}(\gamma>0)$ be the dual space of $\mathscr{H}_{r}$. The norm in $\mathscr{H}_{-r}$ is defined by

$$
\|u\|_{-r}=\sup _{v \in \varkappa_{r}} \frac{\left|(u, v)_{0}\right|}{\|v\|_{r}} \text {. }
$$

It has been shown in [3] that the space $\mathscr{H}_{r}$ is characterized as follows:

$$
\mathscr{H}_{r}\left\{\begin{array}{llrl} 
& =H_{0}^{1}(0,1) \cap H^{2 r}(0,1), & & (1 / 2<\gamma \leq 1) \\
& =H_{0}^{2 r}(0,1), & & (1 / 4<\gamma<1 / 2) \\
& =H^{2 r}(0,1) . & & (-1 / 4<\gamma<1 / 4) .
\end{array}\right.
$$

Let $B(\alpha, \beta)$ be the Banach space of all bounded operators from $\mathscr{H}_{\alpha}$ to $\mathscr{H}_{\beta}$ with the usual operator norm $\mid\|\|_{(\alpha, \beta)}$. When $B$ belongs to $B(\alpha, \alpha)$, in particuler, we write $\|\mid \cdot\|_{\alpha}$ instead of $\mid\|\cdot\| \|_{(\alpha, \alpha)}$.

The following proposition is well-known.

Proposition 3.2. (Interpolation theorem) ([6]). Let $B$ be a bounded operator belonging to $B(\alpha, 0) \cap B(\beta, 0)(\alpha \leq \beta)$. Then, $B$ is the bounded operator belonging to $B(\gamma, 0)(\alpha \leq \gamma \leq \beta)$ and satisfies the following estimate

$$
\|\| B\left\|_{(\gamma, 0)} \leq C(\alpha, \beta, \gamma)\right\| B\|\|_{(\alpha, 0)}^{\beta-\gamma) /(\beta-\alpha)} \mid\|B\|_{(\beta, 0)}^{(\gamma-\alpha) /(\beta-\alpha)} .
$$

where a constant $C(\alpha, \beta, \gamma)$ is independent of $B$.

LEMma 3.1. If $-1 / 4<\gamma \leq 0$, then the operators $A(t)^{-1 / 2} P(t)$ and 
$A(t)^{-1 / 2} P^{*}(t)$ can be extended to bounded operators belonging to $B(\gamma, \gamma)$ with the operator norm independent of $t \in \boldsymbol{R}^{1}$.

Proof. We shall give the proof only for $A(t)^{-1 / 2} P(t)$. Since $C_{0}^{\infty}(0,1)$ is dense in $\mathscr{H}_{r}$ under the norm $\|\cdot\|_{r}$, it is sufficient to show that for any $u \in C_{0}^{\infty}(0,1)$,

$$
\left\|A(t)^{-1 / 2} P(t) u\right\|_{r} \leq C\|u\|_{r} .
$$

Let $u \in C_{0}^{\infty}(0,1)$. Then, by the definition of $\mathscr{H}_{r}$, we have

$$
\begin{aligned}
\left\|A(t)^{-1 / 2} P(t) u\right\|_{r} & =\sup _{v \in \mathscr{P}-r} \frac{\left|\left(A(t)^{-1 / 2} P(t) u, v\right)_{0}\right|}{\|v\|_{-r}} \\
& =\sup _{v \in \boldsymbol{x}_{-r}} \frac{\left|\left(u, P^{*}(t) A(t)^{-1 / 2} v\right)_{0}\right|}{\|v\|_{-r}} \\
& \leq\|u\|_{r} \sup _{v \in \boldsymbol{x}_{-r}} \frac{\left\|P^{*}(t) A(t)^{-1 / 2} v\right\|_{-r}}{\|v\|_{-r}} .
\end{aligned}
$$

We note that $P^{*}(t)$ it a bounded operator from $\mathscr{H}_{1 / 2-\gamma}=H_{0}^{1}(0,1) \cap$ $H^{1-2 r}(0,1)$ to $\mathscr{H}_{-r}=H^{-2 r}(0,1)$ with the operator norm independent of $t$. Hence, we have

$$
\left\|P^{*}(t) A(t)^{-1 / 2} v\right\|_{-r} \leq C\left\|A(t)^{-1 / 2} v\right\|_{1 / 2-r} \leq C\|v\|_{-r},
$$

which together with (3.4) implies our assertion (3.3).

Q.E.D.

\section{§ 4. Main theorem.}

In this section, our main theorem stated in $\S 1$ will be proved by a series of lemmas.

Let $t \in \boldsymbol{R}^{1}$ be fixed. Then, we consider the following differential equation for given $f \in L^{2}(-\infty, \infty ; X)$ and any $h>0$ :

$$
(T(t)+h) u=-\frac{d^{2}}{d x^{2}} u-\frac{d}{d x} P(t) u+P^{*}(t) \frac{d}{d x} u+A(t) u+h u=f .
$$

The solution $u(x)$ is given by

$$
u(x)=(T(t)+h)^{-1} f=R_{t}(h) f=\int_{-\infty}^{\infty} K_{t}(x-s ; h) f(s) d s,
$$

where

(4.2) $\quad K_{t}(x-s ; h)=(2 \pi)^{-1} \int_{-\infty}^{\infty} e^{i(x-s) \xi}\left(\xi^{2}+h+E(t, \xi)\right)^{-1} d \xi, \quad(i=\sqrt{-1})$ 


$$
E(t, \xi)=-i \xi P(t)+i \xi P^{*}(t)+A(t) .
$$

We denote by $R_{t}^{(j)}(h)(=0,1,2, \cdots)$ the operator $\left(\frac{d}{d h}\right)^{j} R_{t}^{(j)}(h)$, which is defined by

$$
R_{t}^{(j)}(h) f=\int_{-\infty}^{\infty} K_{t}^{(j)}(x-s) f(s) d s
$$

where

$$
K_{t}^{(j)}(x-s ; h)=(-1)^{j}(j !)(2 \pi)^{-1} \int_{-\infty}^{\infty} e^{i(x-s) \xi}\left(\xi^{2}+h+E(t, \xi)\right)^{-(j+1)} d \xi .
$$

We often write $R_{t}^{(0)}(h)$ and $K_{t}^{(0)}(x ; h)$ instead of $R_{t}(h)$ and $K_{t}(x ; h)$ respectively.

Let us introduce real-valued $C_{0}$-functions $\varphi(x), \psi(x)$ and $\chi(x)$ defined on $\boldsymbol{R}^{1}$ such that $\varphi(x), \psi(x)$ and $\chi(x)=1$ if $|x| \leq 1,=0$ if $|x| \geq 2$, and that $\varphi(x) \psi(x) \equiv \varphi(x)$ and $\psi(x) \chi(x) \equiv \psi(x)$. For each fixed $t \in \boldsymbol{R}^{1}$ and $\varepsilon>0(\varepsilon<1)$, we denote by $\varphi_{t, \theta}(x)$ the function $\varphi_{t, e}(x)=\varphi((x-t) / \varepsilon)$. Similarly we define the functions $\psi_{t, s}(x)$ and $\chi_{t, o}(x)$. Then, putting $R(h)=(T+h)^{-1}$ and using the resolvent equation, we have

$$
\begin{aligned}
\varphi_{t, \mathrm{e}} R(h)= & \psi_{t, \mathrm{e}} R_{t}(h) \varphi_{t, \mathrm{~s}} \\
& +\psi_{t, \mathrm{e}} R_{t}(h)\left((T(t)+h) \varphi_{t, \mathrm{e}}-\varphi_{t, \mathrm{e}}(T+h)\right) \chi_{t, \mathrm{~s}} R(h) .
\end{aligned}
$$

(We have used that $\varphi_{t, c}(T+h) \chi_{t, c}=\varphi_{t, c}(T+h)$.)

Let $\left\{u_{j}(x)\right\}_{j=1}^{\infty}$ be the normalized eigenfunctions corresponding to the eigenvalues $\left\{\mu_{j}\right\}_{j=1}^{\infty}$ of the operator $T$. Then, by letting (4.6) operate on each $u_{j}(x)$, we have

$$
\begin{aligned}
\left(\mu_{j}+h\right)^{-1} \varphi_{t, \mathrm{~s}} u_{j}= & \psi_{t, \mathrm{~s}} R_{t}(h) \varphi_{t, \mathrm{u}} u_{j} \\
& +\left(\mu_{j}+h\right)^{-1} \psi_{t, \mathrm{e}} R_{t}(h) B(t, s, \varepsilon) u_{j},
\end{aligned}
$$

where we have set

$$
\begin{aligned}
B(t, s, \varepsilon)= & \left(T(t) \varphi_{t, \mathrm{e}}(s)-\varphi_{t, \mathrm{e}}(s) T\right) \chi_{t, \mathrm{c}}(s)=\left(T(t) \varphi_{t, \mathrm{e}}(s)-\varphi_{t, \mathrm{c}}(s) T\right) \\
= & \left(\left(-\frac{d^{2}}{d s^{2}}-\frac{d}{d s} P(t)+P^{*}(t) \frac{d}{d s}+A(t)\right) \varphi_{t, \mathrm{e}}(s)\right. \\
& \left.-\varphi_{t, \mathrm{c}}(s)\left(-\frac{d^{2}}{d s^{2}}-\frac{d}{d s} P(s)+P^{*}(s) \frac{d}{d s}+A(s)\right)\right) .
\end{aligned}
$$

By differentiating (4.7) $n$-times with respect to $h$ in the sense of $L^{2}(-\infty, \infty ; X)$, we have 


$$
\begin{aligned}
(-1)^{n}( & n !)\left(\mu_{j}+h\right)^{-(n+1)} \varphi_{t, \varepsilon} u_{j} \\
& =\psi_{t, \varepsilon} R_{t}^{(n)}(h) \varphi_{t, \varepsilon} u_{j}+\sum_{p=0}^{n} C_{p}\left(\mu_{j}+h\right)^{-(n-p+1)} \psi_{t, \varepsilon} R_{t}^{(p)}(h) B(t, s, \varepsilon) u_{j} .
\end{aligned}
$$

Furthermore, by rewriting (4.9) in the form of the integral equation,

$$
\begin{aligned}
(-1)^{n}(n !)\left(\mu_{j}+h\right)^{-(n+1)} \varphi_{t, s}(x) u_{j}(x) \\
=\psi_{t, \varepsilon}(x) \int_{-\infty}^{\infty} K_{t}^{(n)}(x-s ; h) \varphi_{t, \varepsilon}(s) u_{j}(s) d s \\
\quad+\sum_{p=0}^{n} C_{p}\left(\mu_{j}+h\right)^{-(n-p+1)} \psi_{t, s}(x) \int_{-\infty}^{\infty} K_{t}^{(p)}(x-s ; h) B(t, s, \varepsilon) u_{j}(s) d s \\
=a_{j}(t, x, \varepsilon)+\sum_{p=0}^{n} C_{p} b_{j, p}(t, x, \varepsilon) .
\end{aligned}
$$

We remark that by the regularity theorem for elliptic operators, the eigenfunction $u_{j}(x)$ belongs to $C^{\infty}(-\infty, \infty ; X)$ (the set of smooth functions with values in $X$ ). Hence, the equality (4.10) is well-defined for all $x$. By this fact, we can put $x=t$ in (4.10). Then, we have

$$
\begin{aligned}
& (-1)^{n}(n !)\left(\mu_{j}+h\right)^{-(n+1)} u_{j}(t) \\
& \quad=a_{j}(t, \varepsilon)+\sum_{p=0}^{n} C_{p} b_{j, p}(t, \varepsilon),
\end{aligned}
$$

where we have set $a_{j}(t, \varepsilon)=a_{j}(t, t, \varepsilon)$ and $b_{j}(t, \varepsilon)=b_{j, p}(t, t, \varepsilon)$.

By taking the scalar products in $X$ of both sides of (4.11) and the summation with respect to $j$, and integrating over $(-\infty, \infty)$, we have

$$
\begin{aligned}
(n !)^{2} \sum_{j=1}^{\infty}\left(\mu_{j}+h\right)^{-2(n+1)}= & \sum_{j=1}^{\infty} \int_{-\infty}^{\infty}\left\|a_{\jmath}(t, \varepsilon)\right\|_{0}^{2} d t \\
& +2 \sum_{j=1}^{\infty} \int_{-\infty}^{\infty} \operatorname{Re}\left(a_{j}(t, \varepsilon), \sum_{p=0}^{n} C_{p} b_{j, p}(t, \varepsilon)\right)_{0} d t \\
& +\sum_{j=1}^{\infty} \int_{-\infty}^{\infty}\left\|\sum_{p=0}^{n} C_{p} b_{j, p}(t, \varepsilon)\right\|_{0}^{2} d t .
\end{aligned}
$$

This is our basic equality in proving the main theorem.

From now on, we fix $n$ (integer) such that

$$
n>1 / 2+1 / 2 m-1 \text {. }
$$

Let $\left\{\alpha_{j}(t, \xi)\right\}_{j=1}^{\infty}$ be eigenvalues of the operator $E(t, \xi)$. Then, we have

LEMMA 4.1. For any $r>1 / 2+1 / 2 m$, there exist positive constants $C_{1}(r)$ and $C_{2}(r)$ independent of $h>2$ such that 


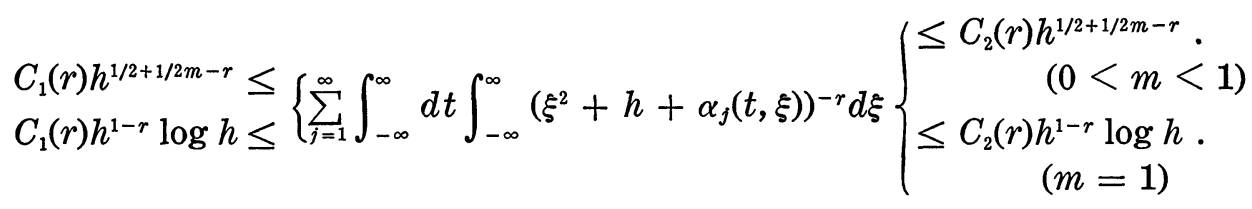

LEMMA 4.2.

$$
\begin{aligned}
\sum_{j=1}^{\infty} \int_{-\infty}^{\infty} d t \int_{-\infty}^{\infty}\left(\xi^{2}+h+\alpha_{j}(t, \xi)\right)^{-2(n+1)} d \xi & \\
& \sim \sum_{j=1}^{\infty} \int_{-\infty}^{\infty} d t \int_{-\infty}^{\infty}\left(\xi^{2}+h+\zeta_{j} q(t)^{-2}\right)^{-2(n+1)} d \xi, \quad \text { as } h \rightarrow \infty
\end{aligned}
$$

The proofs of the above lemmas will be given in this section after the proof of Theorem 1.1. In what follows, we shall state two lemmas concerning the estimates for $\sum_{j=1}^{\infty} \int_{-\infty}^{\infty}\left\|a_{j}(t, \varepsilon)\right\|_{0}^{2} d t$ and $\int_{-\infty}^{\infty} \sum_{j=1}^{\infty}\left\|b_{j, p}(t, \varepsilon)\right\|_{0}^{2} d t$. These lemmas will be proved in the following two sections.

LEMMA 4.3. For any $\varepsilon>0$ and any sufficiently large $r$, there exists a constant $C(r, \varepsilon)$ such that

$$
\begin{aligned}
& \left|\sum_{j=1}^{\infty} \int_{-\infty}^{\infty}\left\|a_{j}(t, \varepsilon)\right\|_{0}^{2} d t-(n !)^{2}(2 \pi)^{-1} \sum_{j=1}^{\infty} \int_{-\infty}^{\infty} d t \int_{-\infty}^{\infty}\left(\xi^{2}+h+\alpha_{j}(t, \xi)\right)^{-2(n+1)} d \xi\right| \\
& \leq C(r, \varepsilon) h^{-r} .
\end{aligned}
$$

LEMMA 4.4. For any sufficiently small $\delta>0$, we can take $\varepsilon(\delta)$ small enough and $h(\delta)$ large enough so that for any $h>h(\delta)$,

$$
\begin{aligned}
\sum_{j=1}^{\infty} \int_{-\infty}^{\infty}\left\|b_{j, p}(t, \varepsilon(\delta))\right\|_{0}^{2} d t & \leq \delta h^{1 / 2+1 / 2 m-2(n+1)} \quad(0<m<1) . \\
( & \left.\leq \delta h^{1-2(n+1)} \log h(m=1) .\right) \quad(0 \leq p \leq n) .
\end{aligned}
$$

Now we shall prove Theorem 1.1.

Proof of Theorem 1.1:

From (4.12) it follows that for any sufficiently small $\delta>0$, there exists a constant $C(\delta)$ such that

$$
\begin{aligned}
\left|(n !)^{2} \sum_{j=1}^{\infty}\left(\mu_{j}+h\right)^{-2(n+1)}-\sum_{j=1}^{\infty} \int_{-\infty}^{\infty}\left\|a_{j}(t, \varepsilon)\right\|_{0}^{2} d t\right| \\
\leq \delta \sum_{j=1}^{\infty} \int_{-\infty}^{\infty}\left\|a_{j}(t, \varepsilon)\right\|_{0}^{2} d t+C(\delta) \sum_{j=1}^{\infty} \int_{-\infty}^{\infty} \sum_{p=0}^{n}\left\|b_{j, p}(t, \varepsilon)\right\|_{0}^{2} d t .
\end{aligned}
$$

Hence, by virtue of Lemmas $4.1,4.3$ and 4.4 , we can first choose $\varepsilon(\delta)$ 
small enough and next $h(\delta)$ large enough so that for any $h>h(\delta)$

$$
\begin{aligned}
\mid \sum_{j=1}^{\infty}\left(\mu_{j}+h\right)^{-2(n+1)} & -\sum_{j=1}^{\infty}(2 \pi)^{-1} \int_{-\infty}^{\infty} d t \int_{-\infty}^{\infty}\left(\xi^{2}+h+\alpha_{j}(t, \xi)\right)^{-2(n+1)} d \xi \mid \\
& \leq \delta h^{1 / 2+1 / 2 m-2(n+1)} \quad(0<m<1) . \\
& \leq \delta h^{1-2(n+1)} \log h \quad(m=1) .
\end{aligned}
$$

Furthermore, by using Lemmas 4.1 and 4.2 , we have

$$
\begin{array}{r}
\sum_{j=1}^{\infty}\left(\mu_{j}+h\right)^{-2(n+1)} \sim(2 \pi)^{-1} \int_{-\infty}^{\infty} d t \int_{-\infty}^{\infty}\left(\xi^{2}+h+\zeta_{j} q(t)^{-2}\right)^{-2(n+1)} d \xi \\
\text { as } h \rightarrow \infty
\end{array}
$$

Now we are in a position to apply the Tauberian theorem due to Keldysh (see [5]) to (4.14). Then, we have

$$
N(h) \sim(\pi)^{-1} \sum_{j=1}^{\infty} \int_{\Omega_{j}(h)}\left(h-\zeta_{j} q(x)^{-2}\right)^{1 / 2} d x \quad \text { as } h \rightarrow \infty,
$$

which completes the proof.

Q.E.D.

Next we shall prove Lemmas 4.1 and 4.2.

Proof of Lemma 4.1:

An argument similar to the proof of Lemma 2.3 shows that for any $u \in \mathscr{D}\left(A_{0}\right)$,

$$
\begin{aligned}
C\left(\left(\xi^{2} u, u\right)_{0}\right. & \left.+\left(1+|t|^{2 m}\right)\left(A_{0} u, u\right)_{0}\right) \\
& \leq\left(\xi^{2} u, u\right)_{0}+(E(t, \xi) u, u)_{0} \\
& \leq C\left(\left(\xi^{2} u, u\right)_{0}+\left(1+|t|^{2 m}\right)\left(A_{0} u, u\right)_{0}\right)
\end{aligned}
$$

Hence, we have

$$
C\left(\xi^{2}+\zeta_{j}\left(1+|t|^{2 m}\right)\right) \leq \xi^{2}+\alpha_{j}(t, \xi) \leq C\left(\xi^{2}+\zeta_{j}\left(1+|t|^{2 m}\right)\right) .
$$

By using (4.15), we have

$$
\begin{aligned}
& \sum_{j=1}^{\infty} \int_{-\infty}^{\infty} d t \int_{-\infty}^{\infty}\left(\xi^{2}+h+\alpha_{j}(t, \xi)\right)^{-r} d \xi \\
& \leq C \sum_{j=1}^{\infty} \zeta_{j}^{-1 / 2 m}\left(\zeta_{j}+h\right)^{1 / 2+1 / 2 m-r} \leq C h^{1 / 2+1 / 2 m-r} \quad(0<m<1) . \\
& \quad\left(\leq C h^{1-r} \log h \quad(m=1) .\right)
\end{aligned}
$$

Thus we have obtained the estimate from above. Similarly we can get the estimate from below.

Q.E.D.

Proof of Lemma 4.2: 
We shall give only an outline. As was remarked in $\S 2$, coefficients of $P(t)$ are uniformly bounded. Hence, for any sufficiently small $\varepsilon>0$, there exists $R(\varepsilon)$ such that for $|t| \geq R(\varepsilon)$ and $u \in \mathscr{D}\left(A_{0}^{1 / 2}\right)$,

$$
\|P(t) u\|_{0} \leq \varepsilon q(t)^{-1}\left\|A_{0}^{1 / 2} u\right\|_{0} .
$$

From (4.16) it readily follows that for $|t| \geq R(\varepsilon)$,

$$
\begin{aligned}
(1-C \varepsilon)\left(\xi^{2}+h+\zeta_{j} q(t)^{-2}\right) & \leq \xi^{2}+h+\alpha_{j}(t, \xi) \\
& \leq(1+C \varepsilon)\left(\xi^{2}+h+\zeta_{j} q(t)^{-2}\right) .
\end{aligned}
$$

On the other hand, for any bounded interval $I$, we have

$$
\sum_{j=1}^{\infty} \int_{I} d t \int_{-\infty}^{\infty}\left(\xi^{2}+h+\alpha_{j}(t, \xi)\right)^{-2(n+1)} d \xi \leq C(I) h^{1-2(n+1)} .
$$

By combining (4.17) and (4.18), we obtain the proof.

Q.E.D.

\section{§5. Proof of Lemma 4.3.}

Lemma 4.3 is proved with the aid of the following lemma.

Lemma 5.1. Let $\alpha$ be any non-negative integer and let $n$ be the fixed integer by (4.13). Then, the operator $\left(\frac{d}{d \xi}\right)^{\alpha}\left(\xi^{2}+h+E(t, \xi)\right)^{-(n+1)}$ belongs to $B_{2}(X)$ and satisfies the following estimate:

$$
\begin{aligned}
&\left\|\left(\frac{d}{d \xi}\right)^{\alpha}\left(\xi^{2}+h+E(t, \xi)\right)^{-(n+1)}\right\|_{2} \\
& \quad \leq C(\alpha, n)\left(\xi^{2}+h\right)^{-\alpha / 2}\left(\sum_{j=1}^{\infty}\left(\xi^{2}+h+\alpha_{j}(t, \xi)\right)^{-2(n+1)}\right)^{1 / 2} .
\end{aligned}
$$

The proof of this lemma will be given at the end of this section

Proof of Lemma 4.3:

By virtue of Proposition 3.1, we have

$$
\begin{aligned}
\sum_{j=1}^{\infty}\left\|a_{j}(t, \varepsilon)\right\|_{0}^{2}= & \int_{-\infty}^{\infty}\left\|K_{t}^{(n)}(t-s ; h) \varphi_{t, \mathrm{e}}(s)\right\|_{2}^{2} d s \\
= & \int_{-\infty}^{\infty}\left\|K_{t}^{(n)}(t-s ; h)\right\|_{2}^{2} d s \\
& +\int_{-\infty}^{\infty}\|\| K_{t}^{(n)}(t-s ; h) \|_{2}^{2}\left(\varphi_{t, \mathrm{e}}(s)^{2}-1\right) d s, \\
= & I(t)+I I(t) .
\end{aligned}
$$


We first investigate the term $I(t)$. By means of the Parseval equality we see that

$$
\begin{aligned}
I(t) & =(n !)^{2}(2 \pi)^{-1} \int_{-\infty}^{\infty}\left\|\left(\xi^{2}+h+E(t, \xi)\right)^{-2(n+1)}\right\|_{2}^{2} d \xi \\
& =(n !)^{2}(2 \pi)^{-1} \sum_{j=1}^{\infty} \int_{-\infty}^{\infty}\left(\xi^{2}+h+\alpha_{j}(t, \xi)\right)^{-2(n+1)} d \xi .
\end{aligned}
$$

Next we shall deal with the term $I I(t)$. We note that

$$
(t-s)^{\alpha} K_{t}^{(n)}(t-s ; h)=C \int_{-\infty}^{\infty} e^{i(t-s) \xi}\left(\frac{d}{d \xi}\right)^{\alpha}\left(\xi^{2}+h+E(t, \xi)\right)^{-2(n+1)} d \xi .
$$

By using Lemma 5.1 and this equality, we calculate as follows:

$$
\begin{aligned}
& \|\| K_{t}^{(n)}(t-s ; h) \|\left.\right|_{2} \\
& \quad \leq C|t-s|^{-\alpha} \int_{-\infty}^{\infty}\left(\xi^{2}+h\right)^{-\alpha / 2}\left(\sum_{j=1}^{\infty}\left(\xi^{2}+h+\alpha_{j}(t, \xi)\right)^{-2(n+1)}\right)^{1 / 2} d \xi, \\
& \quad \leq C|t-s|^{-\alpha} h^{-\alpha / 2+1 / 4}\left(\sum_{j=1}^{\infty} \int\left(\xi^{2}+h+\alpha_{j}(t, \xi)\right)^{-2(n+1)} d \xi\right)^{1 / 2}
\end{aligned}
$$

(Schwarz' inequality)

Furthermore, by virtue of Lemma 4.1 and (5.3), we have

$$
\begin{aligned}
& \int_{-\infty}^{\infty}|I I(t)| d t \leq C(\varepsilon) h^{-\alpha+1 / 2} \sum_{j=1}^{\infty} \int_{-\infty}^{\infty} d t \int_{-\infty}^{\infty}\left(\xi^{2}+h+\alpha_{j}(t, \xi)\right)^{-2(n+1)} d \xi \\
& \leq C(\varepsilon) h^{-\alpha+1+1 / 2 m-2(n+1)} \quad(0<m<1) . \\
& \left(\leq C(\varepsilon) h^{-\alpha+2-2(n+1)} \log h \quad(m=1)\right. \text {.) }
\end{aligned}
$$

(We have used the inequality $\int_{-\infty}^{\infty}|t-s|^{-2 \alpha}\left|\varphi_{t, \varepsilon}(s)^{2}-1\right| d s \leq C(\varepsilon)$.)

By combining (5.2) and (5.4), we get the proof since $\alpha$ is arbitrary.

In order to prove Lemma 5.1, we have to prepare the following two lemmas

LEMMA 5.2. For any non-negative integer $\alpha$, the following estimates hold:

$$
\begin{gathered}
\left\|\left(\frac{d}{d \xi}\right)^{\alpha}\left(\xi^{2}+h+E(t, \xi)\right)^{-1}\right\|_{0} \leq C(\alpha)\left(\xi^{2}+h\right)^{-(\alpha / 2+1)} ; \\
\left\|P(t)\left(\frac{d}{d \xi}\right)^{\alpha}\left(\xi^{2}+h+E(t, \xi)\right)^{-1}\right\|_{0} \leq C(\alpha)\left(\xi^{2}+h\right)^{-(\alpha+1) / 2} .
\end{gathered}
$$

Replacing $P(t)$ by $P^{*}(t)$ in (5.6), we have the same estimate as (5.6). 
Proof. We shall make induction on $\alpha$. It is clear that (5.5) and (5.6) hold when $\alpha=0$. Assuming that (5.5) and (5.6) are valid for $\alpha \leq k$, we shall prove (5.5) when $\alpha=k+1$. For the sake of simplity, we set $\left(\xi^{2}+h+E(t, \xi)\right)^{-1}=F(t, \xi)$. Then, a direct calculation yields

$$
\begin{aligned}
\left(\frac{d}{d \xi}\right)^{(k+1)} F(t, \xi)= & -\left(\frac{d}{d \xi}\right)^{k}\left\{F(t, \xi)\left(2 \xi-i P(t)+i P^{*}(t)\right) F(t, \xi)\right\} \\
= & 2 \xi \sum_{p=0}^{k} C(p)\left(\frac{d}{d \xi}\right)^{p} F(t, \xi)\left(\frac{d}{d \xi}\right)^{(k-p)} F(t, \xi) \\
& +\sum_{q=0}^{k-1} C(q)\left(\frac{d}{d \xi}\right)^{q} F(t, \xi)\left(\frac{d}{d \xi}\right)^{(k-1-q)} F(t, \xi) \\
& +\sum_{r=0}^{k} C(r)\left(\frac{d}{d \xi}\right)^{r} F(t, \xi)\left(-i P(t)+i P^{*}(t)\right) \\
& \cdot\left(\frac{d}{d \xi}\right)^{(k-r)} F(t, \xi)
\end{aligned}
$$

By the assumption of induction, (5.7) implies (5.5) with $\alpha=k+1$. By multipying (5.7) by $P(t)$ from the left, we obtain (5.6) with $\alpha=k+1$.

Q.E.D.

LEMmA 5.3. For any non-negative integer $\alpha,\left(\frac{d}{d \xi}\right)^{\alpha}\left(\xi^{2}+h+E(t, \xi)\right)^{-1}$ belongs to $B_{r}(X)(\gamma \geq 2)$ with the estimate

$$
\begin{aligned}
& \left\|\left(\frac{d}{d \xi}\right)^{\alpha}\left(\xi^{2}+h+E(t, \xi)\right)^{-1}\right\|_{r} \\
& \quad \leq C(\alpha, \gamma)\left(\xi^{2}+h\right)^{-\alpha / 2}\left(\sum_{j=1}^{\infty}\left(\xi^{2}+h+\alpha_{j}(t, \xi)\right)^{-\gamma}\right)^{1 / r} .
\end{aligned}
$$

Proof. As in the proof of the above lemma, we shall give the proof by induction on $\alpha$. It is clear that (5.8) is valid for $\alpha=0$. Under the assumption that (5.8) is true for $\alpha \leq k$, we shall show that (5.8) holds also when $\alpha=k+1$. Noting that if $A \in B_{r}(X)$ and $B \in B(X)$, then $A \cdot B$ belongs to $B_{r}(X)$ with the estimate $\left|\|A \cdot B\|_{r} \leq\right|\|A\|_{r} \mid\|B\|_{0}$, we have, by means of Lemma 5.2 and (5.7), the desired result.

Q.E.D.

Now we shall prove Lemma 5.1.

Proof of Lemma 5.1. As in the proof of Lemma 5.2, we set $F(t, \xi)=$ $\left(\xi^{2}+h+E(t, \xi)\right)^{-1}$. Then, a simple calculation gives 


$$
\begin{aligned}
\left(\frac{d}{d \xi}\right)^{\alpha} F( & t, \xi)^{(n+1)} \\
& =\sum_{\beta_{1}+\beta_{2} \cdots \beta_{n+1}=\alpha} C\left(\beta_{1}, \cdots, \beta_{n+1}\right)\left(\frac{d}{d \xi}\right)^{\beta_{1}} F(t, \xi) \cdots\left(\frac{d}{d \xi}\right)^{\beta_{n+1}} F(t, \xi) .
\end{aligned}
$$

Since each $\left(\frac{d}{d \xi}\right)^{\beta k} F(t, \xi)(k=1,2, \cdots, n+1)$ belongs to $B_{2(n+1)}(X)$, we have, by means of Lemma 5.3 ,

$$
\begin{aligned}
& \left\|\left(\frac{d}{d \xi}\right)^{\alpha} F(t, \xi)^{-(n+1)}\right\| \|_{2} \\
& \quad \leq C(\alpha) \sum_{\beta_{1}+\cdots+\beta_{n+1}=\alpha}\left\|\left(\frac{d}{d \xi}\right)^{\beta_{1}} F(t, \xi)\right\|_{2(n+1)} \cdots\|\|\left(\frac{d}{d \xi}\right)^{\beta_{n+1}} F(t, \xi) \|_{2(n+1)} \\
& \quad \leq C(\alpha)\left(\xi^{2}+h\right)^{-\alpha / 2}\left(\sum_{j=1}^{\infty}\left(\xi^{2}+h+\alpha_{j}(t, \xi)\right)^{-2(n+1)}\right)^{1 / 2} .
\end{aligned}
$$

(We have used the well-known fact that if $A \in B_{p}(X)$ and $B \in B_{q}(X)$, then $A \cdot B \in B_{r}(X)(1 / p+1 / q=1 / r)$ and satisfies $\left.\left|\|A \cdot B\|_{r} \leq C(p, q)\right|\|A\|\left\|_{p} \mid\right\| B \|_{q} \cdot\right)$

Q.E.D.

\section{§ 6. Proof of Lemma 4.4.}

In this section we shall prove Lemma 4.4. For this purpose, recalling the definition of the operator $B(t, s, \varepsilon)$ given by (4.8), we rewrite $B(t, s, \varepsilon)$ as follows:

$$
\begin{aligned}
B(t, s, \varepsilon)= & B_{1}(t, s, \varepsilon)-\frac{d}{d s}(P(t)-P(s)) \varphi_{t, s}(s) \\
& +\frac{d}{d s}\left(P^{*}(t)-P^{*}(s)\right) \varphi_{t, \varepsilon}(s)+(A(t)-A(s)) \varphi_{t, s}(s) \\
= & B_{1}(t, s, \varepsilon)+\sum_{k=0}^{2} H_{k}(t, s, \varepsilon)
\end{aligned}
$$

where

$$
B_{1}(t, s, \varepsilon)=\left[-\left(\frac{d}{d s}\right)^{2}, \varphi_{t, \varepsilon}\right]+\left[\varphi_{t, \varepsilon}, \frac{d}{d s}\right] P(s)+\left[\frac{d}{d s}, P^{*}(s) \varphi_{t, \varepsilon}\right] .
$$

([,] stands for a commutator between two operators.) Then, $b_{j, p}(t, \varepsilon)$ is rewritten as follows:

$$
b_{j, p}(t, \varepsilon)=\left(\mu_{j}+h\right)^{-(n-p+1)} \int_{-\infty}^{\infty} K_{t}^{(p)}(t-s ; h) B_{1}(t, s, \varepsilon) u_{j}(s) d s
$$




$$
\begin{aligned}
& +\left(\mu_{j}+h\right)^{-(n-p+1)} \int_{-\infty}^{\infty} K_{t}^{(p)}(t-s ; h) \sum_{k=0}^{2} H_{k}(t, s, \varepsilon) u_{j}(s) d s \\
= & d_{j, p}(t, \varepsilon)+e_{j, p}(t, \varepsilon)+f_{j, p}(t, \varepsilon)+g_{j, p}(t, \varepsilon) .
\end{aligned}
$$

We note that for $v(s) \in C_{0}^{\infty}(-\infty, \infty ; X)$ (the set of all $X$-valued smooth functions with compact support) and $p \geq 1$,

$$
\int_{-\infty}^{\infty} K_{t}^{(p)}(t-s ; h) \frac{d}{d s} v(s) d s=\int_{-\infty}^{\infty} F_{t}^{(p)}(t-s ; h) v(s) d t
$$

where

$$
F_{t}^{(p)}(t-s ; h)=C \int_{-\infty}^{\infty} e^{i(t-s) \xi} \xi\left(\xi^{2}+h+E(t, \xi)\right)^{-(p+1)} d \xi .
$$

The relation (6.3) is easily obtained with the aid of the vector-valued Fourier transform. The integral (6.4) is valid also for $p=0$. In this case, the integration must be taken in the weak sense. But we don't use this fact below. By virtue of (6.3), we can rewrite $e_{j, p}(t, \varepsilon)(p \geq 1)$ as follows:

$$
e_{j, p}(t, \varepsilon)=\left(\mu_{j}+h\right)^{-(n-p+1)} \int_{-\infty}^{\infty} F_{t}^{(p)}(t-s ; h)(P(s)-P(t)) \varphi_{t, \mathrm{~s}}(s) u_{j}(s) d s .
$$

The following lemma plays an important role in the proof of Lemma 4.4 .

LEMMA 6.1. Let $K_{t}^{(p)}(t-s ; h)$ and $F_{t}^{(p)}(t-s ; h)$ be operators defined by (4.5) and (6.4) respectively. Then, $K_{t}^{(p)}(t-s ; h) A(t), K_{t}^{(p)}(t-s ; h) A(t)^{1 / 2}$ and $F_{t}^{(p)}(t-s ; h) A(t)^{1 / 2}$ can be extended to bounded operators in $X(t \neq s)$ and satisfy the following estimates:

$$
\begin{aligned}
\left\|K_{t}^{(p)}(t-s ; h) A(t)\right\| \|_{0} & \leq C(p, \alpha) h^{-p}|t-s|^{-\alpha}, \\
\left\|\left|K_{t}^{(p)}(t-s ; h) A(t)^{1 / 2}\right|\right\|_{0} & \leq C(p, \alpha) h^{-p-1 / 2}|t-s|^{-\alpha}, \\
& \leq C(p, \beta) h^{-p}|t-s|^{-\beta} .
\end{aligned}
$$

where constants $C(p, \alpha)$ and $C(p, \beta)$ are independent of $t, s$ and $h$, and $\alpha$ and $\beta$ are some constants satisfying $0<\alpha<2$ and $0<\beta<1$ respectively.

Proof. We shall give the proof only for (6.5) with $p=0$, because (6.5) with general $p,(6.6),\left(6.6^{\prime}\right)$ and (6.7) can be proved in the same 
manner. Let $\delta$ be a fixed number such that $0<\delta<1 / 8$. Then, we shall establish the following two assertions:

$$
\left\|K_{t}^{(0)}(t-s ; h) A(t) u\right\|_{0} \leq C(\delta) q(t)^{-(1+2 \delta)}\|u\|_{1 / 2+\delta},
$$

$$
\left\|K_{t}^{(0)}(t-s ; h) A(t) u\right\|_{0} \leq C(\delta) q(t)^{2 \delta}|t-s|^{-2}\|u\|_{-\delta}, \quad \text { for } u \in \mathscr{D}\left(A_{0}\right) \text {. }
$$

All constants $C$ appearing throughout the proof of this lemma may depend only on $\delta$. If we can prove (6.8) and (6.9), the operator $K_{t}^{(0)}(t-s ; h) A(t)$ can be extended to a bounded operator from $\mathscr{H}_{1 / 2+\delta}$ to $X$ and from $\mathscr{H}_{-\delta}$ to $X$ since $\mathscr{D}\left(A_{0}\right)$ is dense in both spaces $\mathscr{H}_{1 / 2+\delta}$ and $\mathscr{H}_{-\delta}$. Hence, the application of the well-known interpolation theorem (Proposition 3.2) shows that

$$
\left.\left\|K_{t}^{(0)}(t-s ; h) A(t)\right\|\right|_{0} \leq C|t-s|^{-\alpha}, \quad(0<\alpha<2) .
$$

Proof of (6.8): Since $\left\|A(t)^{1 / 2+\delta} u\right\|_{0} \leq C q(t)^{-(1+2 \delta)}\left\|A_{0}^{1 / 2+\delta} u\right\|_{0}$ for $u \in \mathscr{D}\left(A_{0}\right)$ by (2.9), it is sufficient to prove that

$$
\left\|K_{t}^{(0)}(t-s ; h) A(t)^{1 / 2-\delta} v\right\|_{0} \leq C\|v\|_{0}, \quad \text { for } v \in \mathscr{D}\left(A_{0}^{1 / 2-\delta}\right) .
$$

By the definition of $K_{t}^{(0)}(t-s ; h)$, we have

$$
K_{t}^{(0)}(t-s ; h) A(t)^{1 / 2-\delta}=C \int_{-\infty}^{\infty} e^{i(t-s) \xi}\left(\xi^{2}+h+E(t, \xi)\right)^{-1} A(t)^{1 / 2-\delta} d \xi
$$

On the other hand, we easily see that

$$
\begin{gathered}
\left\|\left(\xi^{2}+h+E(t, \xi)\right)^{\delta-1 / 2} A(t)^{1 / 2-\delta} v\right\|_{0} \leq C\|v\|_{0}, \\
\|\left(\xi^{2}+h+E(t, \xi)^{-(1 / 2+\delta)}\|\|_{0} \leq C\left(\xi^{2}+1\right)^{-(1 / 2+\delta)} .\right.
\end{gathered}
$$

Hence, in view of (6.11), (6.12) and (6.13), we have (6.10).

Proof of (6.9): Since $\left\|A(t)^{-\delta} u\right\|_{0} \leq C q(t)^{2 \delta}\left\|A_{0}^{-\delta} u\right\|_{0}$ by (2.10), it suffices to show that for $w \in \mathscr{D}\left(A(t)^{1+\delta}\right)$,

$$
\left\|K_{t}^{(0)}(t-s ; h) A(t)^{1+\delta} w\right\|_{0} \leq C|t-s|^{-2}\|w\|_{0} .
$$

The operator $K_{t}^{(0)}(t-s ; h) A(t)^{1+\delta}$ is represented as

$$
\begin{aligned}
K_{t}^{(0)}( & -s ; h) A(t)^{1+\delta} \\
& =C(t-s)^{-2} \int_{-\infty}^{\infty} e^{i(t-s) \xi}\left(\frac{d}{d \xi}\right)^{2}\left(\xi^{2}+h+E(t, \xi)\right)^{-1} A(t)^{1+\delta} d \xi .
\end{aligned}
$$

For brevity, we again put $F(t, \xi)=\left(\xi^{2}+h+E(t, \xi)\right)^{-1}$.

By the resolvent equation, we have 


$$
\begin{aligned}
\left(\frac{d}{d \xi}\right)^{2} F(t, \xi)= & \left(\frac{d}{d \xi}\right)^{2}\left(\xi^{2}+h+A(t)\right)^{-1} \\
& +i\left(\frac{d}{d \xi}\right)^{2}\left\{F(t, \xi) \xi P(t)\left(\xi^{2}+h+A(t)\right)^{-1}\right\} \\
& -i\left(\frac{d}{d \xi}\right)^{2}\left\{F(t, \xi) \xi P^{*}(t)\left(\xi^{2}+h+A(t)\right)^{-1}\right\} \\
= & \mathrm{I}(t, \xi)+i \mathrm{II}(t, \xi)-i \operatorname{III}(t, \xi)
\end{aligned}
$$

By inserting (6.16) into (6.15), we have

$$
\begin{aligned}
K_{t}^{(0)}(t & -s ; h) A(t)^{1+\delta} \\
& \left.=C(t-s)^{-2} \int_{-\infty}^{\infty} e^{i(t-s) \xi}(\mathrm{I}(t, \xi)+i \operatorname{II}(t, \xi))-i \operatorname{III}(t, \xi)\right) A(t)^{1+\delta} d \xi \\
& =C(t-s)^{-2} \sum_{k=1}^{2} K_{t, k}^{(0)}(t-s ; h) A(t)^{1+\delta}
\end{aligned}
$$

It is easy to see that for $w \in \mathscr{D}\left(A(t)^{1+\delta}\right)$,

$$
\left\|\mathrm{I}(t, \xi) A(t)^{1+\delta} w\right\|_{0} \leq C\left(\xi^{2}+1\right)^{-(1-\delta)}\|w\|_{0} .
$$

This implies that

$$
\left\|K_{t, 1}^{(0)}(t-s ; h) A(t)^{1+\delta} w\right\|_{0} \leq\|w\|_{0} .
$$

Next we shall investigate the term II $(t, \xi) A(t)^{1+\delta}$. A simple calculation yields

$$
\begin{aligned}
\mathrm{II}(t, \xi)= & \sum_{\beta_{1}+\beta_{2}=2}\left(\frac{d}{d \xi}\right)^{\beta_{1}}\{F(t, \xi)\} \xi P(t)\left(\frac{d}{d \xi}\right)^{\beta_{2}}\left\{\left(\xi^{2}+h+A(t)\right)^{-1}\right\} \\
& +\sum_{\alpha_{1}+\alpha_{2}=1}\left(\frac{d}{d \xi}\right)^{\alpha_{1}}\{F(t, \xi)\} P(t)\left(\frac{d}{d \xi}\right)^{\alpha_{2}}\left\{\left(\xi^{2}+h+A(t)\right)^{-1}\right\} .
\end{aligned}
$$

We shall consider only the term $\left(\frac{d}{d \xi}\right)\{F(t, \xi)\} P(t)\left(\xi^{2}+h+A(t)\right)^{-1}$, because the other terms can be dealt with in the same way. Putting $2 \xi-i P(t)+i P^{*}(t)=T(t, \xi)$, we have,

$$
\begin{aligned}
& \left(\frac{d}{d \xi}\right)\{F(t, \xi)\} P(t)\left(\xi^{2}+h+A(t)\right)^{-1} \\
& \quad=-F(t, \xi) T(t, \xi) F(t, \xi) P(t)\left(\xi^{2}+h+A(t)\right)^{-1}
\end{aligned}
$$

We shall show that for $w \in \mathscr{D}\left(A(t)^{1+\delta}\right)$,

$$
\left\|F(t, \xi) P(t)\left(\xi^{2}+h+A(t)\right)^{-1} A(t)^{1+\delta} w\right\|_{0} \leq C\left(\xi^{2}+1\right)^{-\delta}\|w\|_{0} .
$$


If we have proved (6.20), then we have

$$
\left\|\left(\frac{d}{d \xi}\right)\{F(t, \xi)\} P(t)\left(\xi^{2}+h+A(t)\right)^{-1} A(t)^{1+\delta} w\right\|_{0} \leq C\left(\xi^{2}+1\right)^{-1 / 2-\delta}\|w\|_{0}
$$

since $\|F(t, \xi) T(t, \xi)\|_{0} \leq C\left(\xi^{2}+1\right)^{-1 / 2}$.

Since the other terms in (6.19) obey the estimate of the same type as (6.21), we see that for $w \in \mathscr{D}\left(A(t)^{1+\delta}\right)$.

$$
\left\|\mathrm{II}(t, \xi) A(t)^{1+\delta} w\right\|_{0} \leq C\left(\xi^{2}+1\right)^{-1 / 2-\delta}\|w\|_{0} .
$$

This implies that

$$
\left\|K_{t, 2}^{(0)}(t-s ; h) A(t)^{1+\delta} w\right\|_{0} \leq C\|w\|_{0} .
$$

Similarly we have

$$
\left\|K_{t, 3}^{(0)}(t-s ; h) A(t)^{1+\delta} w\right\|_{0} \leq C\|w\|_{0} .
$$

Hence, by combining (6.18), (6.22) and (6.23), we have (6.14).

Now we shall prove (6.20). To this end, we rewrite $F(t, \xi) P(t)$ $\left(\xi^{2}+h+A(t)\right)^{-1} A(t)^{1+\delta}$ as follows :

$$
\begin{aligned}
& F(t, \xi) P(t)\left(\xi^{2}+h+A(t)\right)^{-1} A(t)^{1+\delta} \\
& =[F(t, \xi) A(t)]\left[A(t)^{-1} P(t) A(t)^{2 \delta}\right]\left[\left(\xi^{2}+h+A(t)\right)^{-1} A(t)^{1-\delta}\right] \\
& =\mathrm{II}_{1}(t, \xi) \mathrm{II}_{2}(t) \mathrm{II}_{3}(t, \xi) .
\end{aligned}
$$

The operators $\mathrm{II}_{1}(t, \xi)$ and $\mathrm{II}_{3}(t, \xi)$ can be extended to bounded operators in $X$ and satisfy the estimates $\left\|\mathrm{II}_{1}(t, \xi)\right\|_{0} \leq C$ and \|\| $\mathrm{II}_{3}(t, \xi)\|\|_{0} \leq C\left(\xi^{2}+1\right)^{-\delta}$ respectively. Hence, in order to prove (6.20), it is sufficient to show that $\mathrm{II}_{2}(t)$ can be extended to a bounded operator in $X$ and that $\left\|\mathrm{II}_{2}(t)\right\|_{0} \leq C$. But this fact readily follows from Lemma 3.1. In fact, we have for $w \in \mathscr{D}\left(A(t)^{2 \delta}\right)$,

$$
\begin{aligned}
& \left\|A(t)^{-1} P(t) A(t)^{2 \delta} w\right\|_{0} \\
& \quad \leq C\left\|A(t)^{-2 \delta} A(t)^{-1 / 2} P(t) A(t)^{2 \delta} w\right\|_{0} \\
& \quad \leq C q(t)^{2 \delta}\left\|A(t)^{-1 / 2} P(t) A(t)^{2 \delta} w\right\|_{-2 \delta} \\
& \quad \leq C q(t)^{2 \delta}\left\|A(t)^{2 \delta} w\right\|_{-2 \delta} \leq C\|w\|_{0},
\end{aligned}
$$

which implies that $\left\|\mathrm{II}_{2}(t)\right\|_{0} \leq C$ since $\mathscr{D}\left(A(t)^{2 \delta}\right)$ is dense in $X$. Q.E.D.

LEMMA 6.2. Let $g_{j, p}(t, \varepsilon)(j=1,2, \cdots, p=0,1, \cdots, n)$ be the functions defined in (6.2). Then, for any sufficiently small $\delta>0$, there exists $\varepsilon(\delta)$ such that for $\varepsilon<\varepsilon(\delta)$, 


$$
\begin{aligned}
\sum_{j=1}^{\infty} \int_{-\infty}^{\infty}\left\|g_{j, p}(t, \varepsilon)\right\|_{0}^{2} d t & \leq \delta h^{1 / 2+1 / 2 m-2(n+1)} & & (0<m<1) \\
( & \leq \delta h^{1-2(n+1)} \log h & & (m=1) .)
\end{aligned}
$$

Here we should note that $\varepsilon(\delta)$ is taken independently of $h>2$.

Proof. We shall consider only the case of $0<m<1$. Two different methods of estimates will be employed in proving this lemma.

Case $1,0 \leq p<n+1-1 / 4-1 / 4 m$ : By the definition of $g_{j, p}(t, \varepsilon)$, we have

$$
\begin{aligned}
& \sum_{j=1}^{\infty} \int_{-\infty}^{\infty}\left\|g_{j, p}(t, \varepsilon)\right\|_{0}^{2} d t \\
& \quad \leq \sum_{j=1}^{\infty}\left(\mu_{j}+h\right)^{-2(n-p+1)} \int_{-\infty}^{\infty} d t\left(\int_{-\infty}^{\infty}\left\|K_{t}^{(p)}(t-s ; h) H_{2}(t, s, \varepsilon) u_{j}(s)\right\|_{0} d s\right)^{2} .
\end{aligned}
$$

Set I $(t, s, j)=\left\|K_{t}^{(p)}(t-s ; h) H_{2}(t, s, \varepsilon) u_{j}(s)\right\|_{0}$. Then, by virtue of Lemma 6.1 and (2.14) in Lemma 2.2, I $(t, s, j)$ is estimated as follows:

$$
\begin{array}{rlrl}
I(t, s, j) & =\left\|\left[K_{t}^{(p)}(t-s ; h) A(t)\right]\left[A(t)^{-1}(A(t)-A(s)) \varphi_{t, c}(s)\right] u_{j}(s)\right\|_{0} \\
& \leq C h^{-p}|t-s|^{1-\alpha}\left\|u_{j}(s)\right\|_{0}, & \text { for }|t-s| \leq 2 \varepsilon . \\
( & =0 & & \text { for }|t-s|>2 \varepsilon)
\end{array}
$$

Hence, it follows that

$$
\begin{aligned}
\int_{-\infty}^{\infty} d t\left(\int_{-\infty}^{\infty} \mathrm{I}(t, s, j) d s\right)^{2} & \leq C h^{-2 p} \int_{-\infty}^{\infty} d t\left(\int_{|t-s| \leq 2 \varepsilon}|t-s|^{1-\alpha}\left\|u_{j}(s)\right\|_{0} d s\right)^{2} \\
& \leq C h^{-2 p} \int_{|s| \leq 2 \varepsilon}|s|^{1-\alpha} d s \int_{|r| \leq 2 \varepsilon}|r|^{1-\alpha} d r \\
& \cdot \int_{-\infty}^{\infty}\left\|u_{j}(t+s)\right\|_{0}\left\|u_{j}(t+r)\right\|_{0} d t \\
\leq & C h^{-2 p} \varepsilon^{4-2 \alpha}
\end{aligned}
$$

(We have used that $0<\alpha<2$ and $\int_{-\infty}^{\infty}\left\|u_{j}(t+s)\right\|_{0}\left\|u_{j}(t+r)\right\|_{0} d t \leq 1$ (Schwarz's inequality).) On the other hand, we have proved in Proposition 2.1 that if $2(n-p+1)>1 / 2+1 / 2 m$,

$$
\sum_{j=1}^{\infty}\left(\mu_{j}+h\right)^{-2(n-p+1)} \leq C h^{1 / 2+1 / 2 m-2(n-p+1)} .
$$

Hence, by combining (6.24) and (6.25), we have the desired estimate. 
Case $2, n \geq p \geq n+1-1 / 4-1 / 4 m>(1 / 4+1 / 4 m): \quad\left(m \leq 1 / 3^{1)}\right.$

It is easily seen that the operator $K_{t}^{(p)}(t-s ; h) H_{2}(t, s, \varepsilon)$ belongs to $B_{2}(X)$ since $\left(\xi^{2}+h+E(t, \xi)\right)^{-1} A(t)$ can be extended to a bounded operator in $X$. Therefore, by virtue of Proposition 3.1, we have

$$
\begin{aligned}
\sum_{j=1}^{\infty}\left\|g_{j, p}(t, \xi)\right\|_{0}^{2} & \leq h^{-2(n-p+1)} \sum_{j=1}^{\infty}\left\|\int_{-\infty}^{\infty} K_{t}^{(p)}(t-s ; h) H_{2}(t, s, \varepsilon) u_{j}(s) d s\right\|_{0}^{2} \\
& =h^{-2(n-p+1)} \int_{-\infty}^{\infty}\left\|K_{t}^{(p)}(t-s ; h) H_{2}(t, s, \varepsilon)\right\| \|_{2}^{2} d s .
\end{aligned}
$$

Furthermore, it follows from (2.14) in Lemma 2.2 that

$$
\begin{aligned}
\| K_{t}^{(p)}(t & -s ; h) H_{2}(t, s, \varepsilon) \|_{2} \\
& =\left\|\left[K_{t}^{(p)}(t-s ; h) A(t)\right]\left[A(t)^{-1}(A(t)-A(s)) \varphi_{t, c}(s)\right]\right\|_{2} \\
& \leq C \varepsilon\left\|K_{t}^{(p)}(t-s ; h) A(t)\right\| \|_{2} .
\end{aligned}
$$

On the other hand, with the aid of the Parseval equality we have

$$
\begin{gathered}
\int_{-\infty}^{\infty}\left\|K_{t}^{(p)}(t-s ; h) A(t)\right\|_{2}^{2} d t \\
=C \int_{-\infty}^{\infty}\|\|\left(\xi^{2}+h+E(t, \xi)\right)^{-(p+1)} A(t) \|_{2}^{2} d \xi \\
\leq C \int_{-\infty}^{\infty}\|\|\left(\xi^{2}+h+E(t, \xi)\right)^{-p} \|_{2}^{2} d \xi \\
=C \sum_{j=1}^{\infty} \int_{-\infty}^{\infty}\left(\xi^{2}+h+\alpha_{j}(t, \xi)\right)^{-2 p} d \xi .
\end{gathered}
$$

Hence, by combinig (6.27) and (6.28) with (6.26), we have

$$
\sum_{j=1}^{\infty}\left\|g_{j, p}(t, \varepsilon)\right\|_{0}^{2} \leq C \varepsilon h^{-2(n-p+1)} \sum_{j=1}^{\infty} \int_{-\infty}^{\infty}\left(\xi^{2}+h+\alpha_{j}(t, \xi)\right)^{-2 p} d \xi,
$$

which together with Lemma 4.1 implies that

$$
\left.\sum_{j=1}^{\infty} \int_{-\infty}^{\infty}\left\|g_{j, p}(t, \varepsilon)\right\|_{0}^{2} d t \leq C \varepsilon h^{1 / 2+1 / 2 m-2(n+1)}\right) .
$$

This completes the proof.

Q.E.D.

LEMMA 6.3. Let $e_{j, p}(t, \varepsilon)$ be the function defined in (6.2). Then, for any sufficiently small $\delta>0$, there exist $\varepsilon(\delta)$ and $h(\delta)$ such that for $h>h(\delta)$,

$$
\begin{aligned}
\sum_{j=1}^{\infty} \int_{-\infty}^{\infty}\left\|e_{j, p}(t, \varepsilon(\delta))\right\|_{0}^{2} d t & \leq \delta h^{1 / 2+1 / 2 m-2(n+1)} & & (0<m<1), \\
& \left(\leq \delta h^{1-2(n+1)} \log h\right. & & (m=1 .)
\end{aligned}
$$

1) If $m>1 / 3$, it is enough to consider only the case 1 . 
Proof.

Case $1, n \geq p \geq 1$ :

By using (6.7) instead of (6.5), the proof is obtained exactly in the same way as in the proof of Lemma 6.2.

Case 2, $p=0$ :

Recalling the definition of $H_{0}(t, s, \varepsilon)$ given by (6.1), we rewrite $H_{0}(t, s, \varepsilon)$ as follows :

$$
\begin{aligned}
H_{0}(t, s, \varepsilon) & =-(P(t)-P(s)) \varphi_{t, s}(s) \frac{d}{d s}-(P(t)-P(s)) \varphi_{t, s}^{\prime}(s)+P^{\prime}(s) \varphi_{t, \varepsilon}(s) \\
& =\mathrm{I}_{1}(t, s, \varepsilon)+\mathrm{I}_{2}(t, s, \varepsilon)+\mathrm{I}_{3}(t, s, \varepsilon),
\end{aligned}
$$

where we put $P^{\prime}(s)=\frac{d}{d s} P(s)$ and $\varphi_{t, \mathrm{~s}}^{\prime}(s)=\frac{d}{d s} \varphi_{t, \mathrm{~s}}(s)$. Then, $e_{j, 0}(t, \varepsilon)$ is rewritten as follows:

$$
\begin{aligned}
e_{j, 0}(t, \varepsilon) & =\left(\mu_{j}+h\right)^{-(n+1)} \int_{-\infty}^{\infty} K_{t}^{(0)}(t-s ; h) \sum_{k=1}^{3} \mathrm{I}_{k}(t, s, \varepsilon) u_{j}(s) d s \\
& =e_{j, 0,1}(t, \varepsilon)+e_{j, 0,2}(t, \varepsilon)+e_{j, 0,3}(t, \varepsilon) .
\end{aligned}
$$

(1) Case 2-1, estimate of $\sum_{j=1}^{\infty} \int_{-\infty}^{\infty}\left\|e_{j, 0,1}(t, \varepsilon)\right\|_{0}^{2} d t$ :

We note that there exists a constant $C$ independent of $j$ such that

$$
\left\|\frac{d}{d s} u_{j}(s)\right\|_{0}^{2} d s \leq C \mu_{j} \text {. }
$$

Put $\mathrm{II}_{1}(t, s, j)=\left\|K_{t}^{(0)}(t-s ; h) \mathrm{I}_{1}(t, s, \varepsilon) u_{j}(s)\right\|_{0}$. Then, an argument similar to the proof of the case 1 in Lemma 6.2 shows that

$$
\begin{array}{rlrl}
\mathrm{II}_{1}(t, s, j) & \leq C h^{-1 / 2}|t-s|^{1-\alpha}\left\|\frac{d}{d s} u_{\jmath}(s)\right\|_{0}, & & \text { for }|t-s| \leq 2 \varepsilon . \\
( & =0 \quad & \text { for }|t-s|>2 \varepsilon,)
\end{array}
$$

Furthermore, by using (6.29) and this estimate, we obtain

$$
\int_{-\infty}^{\infty} d t\left(\int_{-\infty}^{\infty} \mathrm{II}_{1}(t, s, j) d s\right)^{2} \leq C h^{-1} \varepsilon^{4-2 \alpha} \mu_{j} .
$$

Hence, by virtue of Proposition 2.1, it readily follows that

$$
\begin{aligned}
\sum_{j=1}^{\infty} \int_{-\infty}^{\infty}\left\|e_{j, 0,1}(t, \varepsilon)\right\|_{0}^{2} d t & \leq C h^{-1} \varepsilon^{4-2 \alpha} \sum_{j=1}^{\infty}\left(\mu_{j}+h\right)^{-2(n+1)} \mu_{j} \\
& \leq C h^{-1} \varepsilon^{4-2 \alpha} h^{1 / 2+1 / 2 m-2 n-1} .
\end{aligned}
$$


Choosing $\varepsilon(\delta)$ in the above estimate so that $C \varepsilon^{4-2 \alpha}<\delta$, we can get the desired estimate for the term $\sum_{j=1}^{\infty} \int_{-\infty}^{\infty}\left\|e_{j, 0,1}(t, \varepsilon)\right\|_{0}^{2} d t$.

(2) Case 2-2, estimate of $\sum_{j=1}^{\infty} \int_{-\infty}^{\infty}\left\|e_{j, 0,2}(t, \varepsilon)\right\|_{0}^{2} d t$ :

Set $\operatorname{II}_{2}(t, s, j)=\left\|K_{t}^{(0)}(t-s ; h) \mathrm{I}_{2}(t, s, \varepsilon) u_{j}(s)\right\|_{0}$. Then, by virtue of (6.6) in Lemma 6.1, we have

$$
\begin{array}{rlrl}
\mathrm{II}_{2}(t, s, j) & \leq C(\varepsilon) h^{-1 / 2}|t-s|^{1-\alpha}\left\|u_{j}(s)\right\|_{0}, & & \text { for }|t-s| \leq 2 \varepsilon . \\
(=0 & & \text { for }|t-s| \geq 2 \varepsilon .)
\end{array}
$$

Hence, as in the proof of the above case $2-1$, we have

$$
\sum_{j=1}^{\infty} \int_{-\infty}^{\infty}\left\|e_{j, 0,2}(t, \varepsilon)\right\|_{0}^{2} d t \leq C(\varepsilon) \cdot h^{-1} h^{1 / 2+1 / 2 m-2(n+1)} .
$$

Choosing $h$ such that $C(\varepsilon) h^{-1}<\delta$, we have have the desired estimate for the term $\sum_{j=1}^{\infty} \int_{-\infty}^{\infty}\left\|e_{j, 0,2}(t, \varepsilon)\right\|_{0}^{2} d t$.

Similarly we can see that $\sum_{j=1}^{\infty} \int_{-\infty}^{\infty}\left\|e_{j, 0,3}(t, \varepsilon)\right\|_{0}^{2} d t$ is estimated as in $\sum_{j=1}^{\infty} \int_{-\infty}^{\infty}\left\|e_{j, 0,2}(t, \varepsilon)\right\|_{0}^{2} d t$.

Combining the results of the cases $2-1$ and 2-2, the proof is completed.

Q.E.D.

A method similar to those given in the proofs of Lemmas 6.2 and 6.3 can be applied also to $d_{j, p}(t, \varepsilon)$ and $f_{j, p}(t, \varepsilon)$. Thus the proof of Lemma 4.4 is completed.

\section{Generalizations.}

The method developed in the preceding sections can be applied to more general problems.

7.1. Multi-dimensional case.

Let us consider the following problem:

$$
-\sum_{j=1}^{k} \frac{\partial^{2}}{\partial x_{j}^{2}} u-\Delta_{y} u=\lambda u, \quad u \in H_{0}^{1}(\Omega),
$$

where $\Delta_{y}=\sum_{j=1}^{n} \frac{\partial^{2}}{\partial y_{j}^{2}}$.

Here we impose the following assumptions on a domain $\Omega$ in $\boldsymbol{R}^{n+k}$. 
(A-1) $\Omega$ is a domain of the form $\Omega=\left\{(x, y) \mid x \in R^{k}, y \in \Omega(x) \subset R^{n}\right\}$, where $\Omega(x)$ is a bounded domain for each fixed $x \in R^{k}$.

(A-2) There exists a family of differentiable mappings of class $C^{\infty}$, $\left\{g(x, y)=\left(g_{i}(x, y)\right)_{i=1}^{n}\right\}$, from $\Omega(x)$ onto $\Omega(0)$ satisfying the following assumptions :

Let $m$ be a positive constant such that $0<m \leq k / n$ and let $C$ be a positive constant independent of $x, y \in \Omega(x)$ and $\xi \in \boldsymbol{R}^{n}$.

(1) For the Jacobian $J(x, y)=\left|\operatorname{det}\left(\frac{\partial}{\partial y_{j}} g_{i}(x, y)\right)\right|$,

$$
C(1+|x|)^{m n} \leq J(x, y) \leq C(1+|x|)^{m n} .
$$

(2) For $g_{i j}(x, y)=\frac{\partial}{\partial y_{i}} g_{i}(x, y)$,

$$
C(1+|x|)^{m}|\xi|^{2} \leq\left(\sum_{i, j=1}^{n} g_{i j}(x, y) \xi_{i}\right)^{2} \leq C(1+|x|)^{m}|\xi|^{2}
$$

(3) For any multi-index $\alpha$ with $|\alpha| \leq 2,\left|\left(\frac{\partial}{\partial x}\right)^{\alpha} g_{i}(x, y)\right| \leq C(1+|x|)^{m-|\alpha|}$.

If a domain $\Omega$ satisfies the above assumptions (A-1) and (A-2), we say that $\Omega$ belongs to $D(m)$.

THEOREM 7.1. Let $\Omega$ be a domain belonging to $D(m)$ with $0<m \leq k / n$. Let $\left\{\alpha_{j}(x)\right\}_{j=1}^{\infty}$ be eigenvalues of the operator $-\Delta_{y}$ with the domain of definition $\mathscr{D}\left(\Delta_{y}\right)=H_{0}^{1}(\Omega(x)) \cap H^{2}(\Omega(x))$. $N(h)$ denotes the number of eigenvalues less than $h$ of the problem (7.1). Then,

$$
N(h) \sim C \sum_{j=1}^{\infty} \int_{\Omega_{j}(h)}\left(h-\alpha_{j}(x)\right)^{k / 2} d x \quad \text { as } h \rightarrow \infty,
$$

where $C=\left((2 \sqrt{\pi})^{k} \Gamma(1+k / 2)\right)^{-1}$ and $\Omega_{j}(h)=\left\{x \in \boldsymbol{R}^{K} \mid \alpha_{j}(x)>h\right\}$.

7.2. Case of domains with a finite number of holes:

Consider the following eigenvalue problem:

$$
-\frac{\partial^{2}}{\partial x^{2}} u-\frac{\partial^{2}}{\partial y^{2}} u=\lambda u, \quad u \in H_{0}^{1}(\Omega)
$$

Here we assume that an open domain $\Omega=\{(x, y) \mid-\infty<x<\infty, y \in \Omega(x)\}$ with the smooth boundary is decomposed into

$$
(-\infty,-R) \times(0, q(x)) \cup \Omega_{0} \cup(R, \infty) \times(0, q(x)),
$$


where $R$ is some constant and $q(x)$ is a smooth function belonging to $K(m)$, while $\Omega_{0}$ is not necessarily a simply connected domain but may have a finite number of holes.

THEOREM 7.2. Let $\Omega$ be an open domain satisfying the above assumptions. Let $\left\{\alpha_{j}(x)\right\}_{j=1}^{\infty}$ be eigenvalues of the operator $-\frac{\partial^{2}}{\partial y^{2}}$ with the domain of definition $H_{0}^{1}(\Omega(x)) \cap H^{2}(\Omega(x))$. Then,

$$
N(h) \sim(\pi)^{-1} \int_{\Omega_{j}(h)}\left(h-\alpha_{j}(x)\right)^{1 / 2} d x \quad \text { as } h \rightarrow \infty .
$$

\section{REFERENCES}

[1] C. Clark, An asymptotic formula for the eigenvalues of the Laplace operator in an unbounded domain, Bull. Amer. Math. Soc., 72 (1966), pp. 709-713.

[2] I. M. Glazman and B. Ya. Skacek, On the discrete part of the spectrum of the Laplacian in limit cylindrical domains, Dok. Akad. Nauk SSSR, 147 (1962), pp. $760-763$.

[3] P. Grisvard, Caractérisation de quelques espaces d'interpolation, Ark. Rat. Mech. Anal., 25 (1967), pp. 40-63.

[4] D. E. Hewgill, On the eigenvalues of the Laplacian operator in an unbounded domain, Arch. Rational Mech. Anal., 27 (1967), pp. 153-164.

[5] A. G. Kostjuchenko and B. M. Levitan, Asymptotic behavior of the eigenvalues of the Sturm-Liouville operator problem, Funkcional Anal. i Prilozen, 1 (1967), pp. 86-96 (in Russian), Funct. Analysis Appl., 1 (1967), pp. 75-83.

[ 6 ] J. L. Lions and E. Magenes, Problèmes aux limites non homogénes et applications, 1, Paris Dunod (1968).

[ 7] E. C. Titchmarsh, Eigenfunction Expansions associated with second order differential equations, vol. II. Oxford University Press (1958).

Department of Mathematics

Nagoya University 